\title{
Design and simulation of an adaptive beam smart antenna using MATLAB
}

\author{
Aaron Don M. Africa, Rica Rizabel M. Tagabuhin, Jan Jayson S. D. Tirados \\ Department of Electronics and Communications Engineering, De La Salle University, Manila, Philippines
}

\begin{tabular}{|c|c|}
\hline Article Info & ABSTRACT \\
\hline Article history: & \multirow{10}{*}{$\begin{array}{l}\text { Signals transmitted over a long range of distance may pass through several } \\
\text { obstacles and scatter, taking multiple paths to reach the receiver. } \\
\text { Beamforming antennas are controlled electronically to adjust the radiation } \\
\text { pattern following the first received signal. This allows the antenna to } \\
\text { maximize the received signal and consequently, suppress the interfering } \\
\text { signals received. A smart antenna should be able to diminish noise, increase } \\
\text { the signal to noise ratio, and have better system competence. The adaptive } \\
\text { beam makes use of the spacing of the several antennas and the phase of the } \\
\text { signal of each antenna array to control the shape and direction of the signal } \\
\text { beam. This paper focuses on the use of smart antennas using an adaptive } \\
\text { beam method as a better system for the transmission of signals. A simulation } \\
\text { between the existing Omnidirectional antenna system and the smart antenna } \\
\text { system will be made and compared. The paper will discuss the corresponding } \\
\text { advantages that a smart antenna system has compared to the Omnidirectional } \\
\text { antenna system. }\end{array}$} \\
\hline Received Sep 6, 2020 & \\
\hline Revised Nov 19, 2020 & \\
\hline Accepted Nov 30, 2020 & \\
\hline Keywords: & \\
\hline Beamforming & \\
\hline MATLAB & \\
\hline Omnidirectional antenna & \\
\hline Sensor array analyzer & \\
\hline Smart antenna & \\
\hline
\end{tabular}

This is an open access article under the $\underline{C C B Y-S A}$ license.

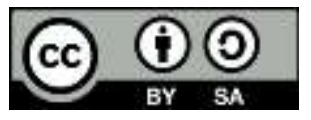

\section{Corresponding Author:}

Aaron Don M. Africa

Department of Electronics and Communications Engineering

De La Salle University, Manila

2401 Taft Ave., Malate, Manila 1004, Philippines

Email: aaron.africa@dlsu.edu.ph

\section{INTRODUCTION}

Antennas are devices that send and receive electromagnetic waves over a long distance. A transmitter antenna takes physical energy and converts it into electrical energy and into radio waves that can travel miles around the Earth or towards the atmosphere and back to Earth. A receiver antenna obtains the radio waves, turns it back to electrical signals, and into a physical form. Different antennas are used to cater to different requirements [1]. In antenna design, three fundamental properties should always be considered: Gain, Polarization, and Direction [2]. Gain is the increase of energy of the radio waves by the antenna. It is the ratio of the power received and the power transmitted. The measurement $\mathrm{dBi}$ is used when the gain is compared to an isotropic antenna, an ideal, lossless, antenna that radiates equally in all directions with a gain equal to 1.0. The measurement $\mathrm{dBd}$ is used when the gain is compared to an ideal lossless half-wave antenna, whose gain is equal to $2.15 \mathrm{~dB}$.

The polarization is the orientation of the transmitting signal of the antenna. Linear polarization is when the electric field and the magnetic field are constricted in one plane and travels in one direction. Elliptical or Circular polarization is when the electric field and the magnetic field rotates as the signal propagates. The direction, or directivity, is the shape of the radiation pattern. The radiation pattern of an antenna shows the correlation of the power radiated by the antenna with its direction. An antenna can be classified based on its directivity. An omnidirectional antenna radiates energy in all directions. The gain of an 
omnidirectional antenna is smaller in comparison to a directional antenna [3]. Directional antennas use different methods to focus on the direction of the radiated energy in a particular direction. The longer distance can be covered by the radio waves radiated by directional antennas [4].

\section{BACKGROUND OF THE STUDY}

An omnidirectional antenna gives off a 360-degree horizontal radiation pattern and is usually used when all directions need to be covered in varying degrees of vertical coverage. The radio power covered by this type of antenna is equal in all directions that are perpendicular to an axis. These omnidirectional antennas are fairly easy to install and due to its shape, are also easy to attach. The main disadvantage of this type of antenna is that coverage under the antenna is poor [5]. Recent research topics that are done due to improvements and advantages over the omnidirectional systems are about smart antenna systems. A smart antenna system is composed of a group of antennas that have smart processing algorithms that can be used for the identification of spatial signal signatures. There are two types of smart antennas which are the switched beam systems and the adaptive array systems. The creation of a radiation pattern of an antenna array in which signals at certain angles create constructive interference while simultaneously creating a destructive interference in other angles is a method called beamforming which is used in the adaptive array systems. Beamforming, when executed correctly, allows a signal to be focused on a direction where you want it to go and is a method that the smart antenna system utilizes. Rather than radiating a signal in all directions, using the beamforming method allows a higher signal quality to be achieved without the need for a boost in broadcasting power. Though this may be the case, using a beamforming method requires high processing and efficiency which may negate the aforementioned advantages if the requirements are not met. With the development of technology and the continuing research on wireless communication, experimenting on the smart antenna system, which uses spatial processing, is one of the best things to explore.

\section{STATEMENT OF THE PROBLEM}

In radio communications, the omnidirectional antenna is used to broadcast signals from one point to another. With the use of this antenna, power is dissipated equally in all directions that are perpendicular to an axis. Though this may be useful in simple RF environments, the omnidirectional antenna scatters the signal in such a way that only a small percentage of the energy is delivered to its users due to scattering the signal in an unfocused manner. This shows an obvious limitation in the dispersion of the signal. To be able to utilize the distribution of a signal more effectively, a smart antenna system is to be used. The smart antenna system can dynamically react to the environment that it is placed in which in turn provides a signal and frequency that is better suited for communications. Though there are various methods in the implementation of a smart antenna system, we focus on the beamforming method as the main process for the smart antenna system.

\section{SIGNIFICANCE OF THE STUDY}

In wireless communications, it is evident that there are limitations that need to be overcome. As the omnidirectional antenna has various problems that direct to the capacity, radio propagation environment, etc., the smart antenna system is proposed to solve the problems stated. The smart antenna system solves the problems by increasing the coverage and capacity of the system and by applying directional findings. This further improves the wireless communication system that is being commercially used. With the comparison of an omnidirectional antenna to the smart antenna system, the study aims to show the advantages of using the smart antenna system in implementation rather than using a simple omnidirectional antenna. With a broader knowledge of information regarding smart antenna systems, many other applications may be utilized with a better understanding of the proposed system. The study will also serve to give a better understanding of what spatial processing and beamforming do concerning a smart antenna system. The study also aims to help readers be able to simulate an omnidirectional antenna and a smart antenna system to be able to variate the values for comparing and contrasting performance. This will also serve as a great reference for introductory information to the basic types of antennas that are available with the current technology.

\section{DESCRIPTION OF THE SYSTEM}

\subsection{Omnidirectional antenna parameters}

Array Geometry: Linear, Number of elements $=2$, Spacing Element $=5 \mathrm{~m}$, Element: Isotropic Antenna, Propagation Speed $=3 \times 108 \mathrm{~m} / \mathrm{s}$, Signal Frequency $=300 \mathrm{MHz}$. Figure 1 shows the Array Geometry of the omnidirectional antenna. 


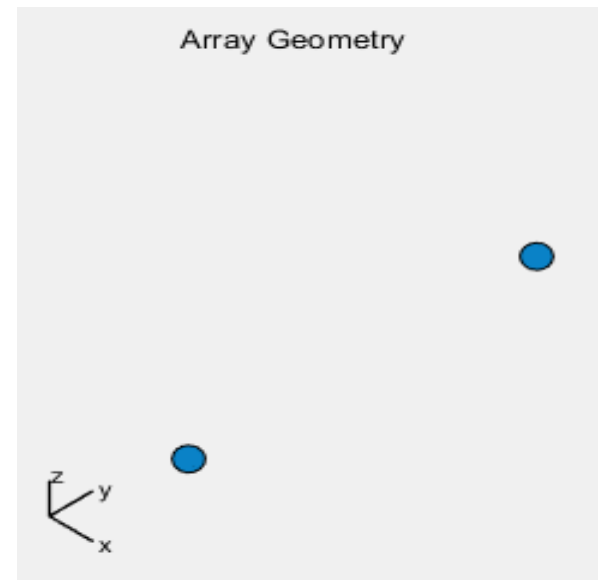

Figure 1. Array geometry of the omnidirectional antenna

\subsection{Adaptive beamforming smart parameters}

Array geometry: circular planar, radius $=2 \mathrm{~m}$, spacing element $=0.5 \mathrm{~m}$, element: Cosine antenna, propagation speed $=3 \times 108 \mathrm{~m} / \mathrm{s}$, signal frequency $=300 \mathrm{MHz}$. Figure 2 shows the array geometry of the smart antenna.

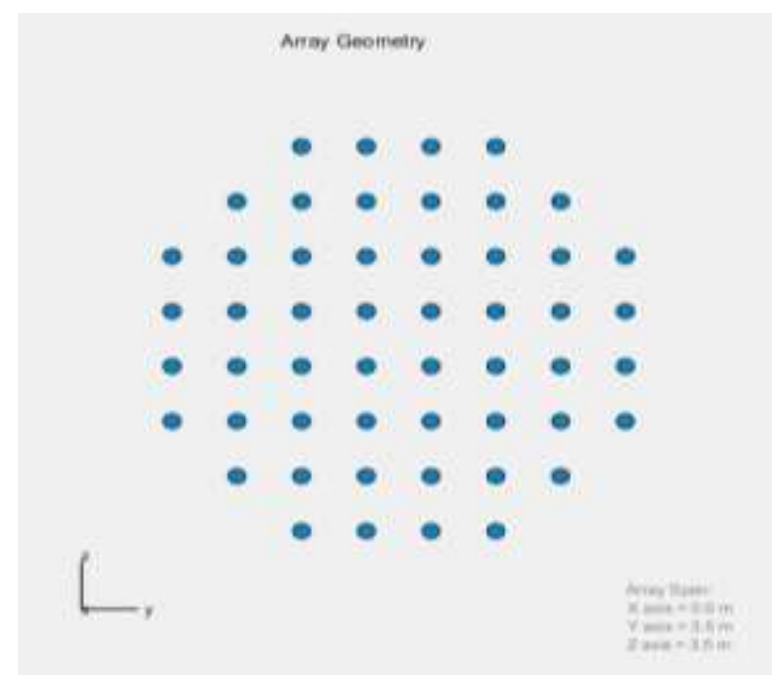

Figure 2. Array geometry of the smart antenna

\section{METHODOLOGY}

The application used to simulate the omnidirectional antenna and the adaptive beamforming smart antenna is the sensor array analyzer application in MATLAB. A phased array system toolbox add-on was installed to access the application. The application requires the user to input certain parameters to simulate the antenna. For the omnidirectional antenna, a linear array geometry was selected, the number of elements was 2 , the spacing element was set to $5 \mathrm{~m}$, the chosen antenna element was an isotropic antenna, the propagation speed was set to $3 \times 108 \mathrm{~m} / \mathrm{s}$ and the signal frequency was set to $300 \mathrm{MHz}$. For the adaptive beamforming smart antenna, the array geometry chosen was a circular plane with the radius set to $5 \mathrm{~m}$, the spacing element was set to $0.5 \mathrm{~m}$, the chosen antenna element was a cosine antenna element, the speed propagation was set to $3 \times 108 \mathrm{~m} / \mathrm{s}$, and signal frequency was set to $300 \mathrm{MHz}$. To be able to compare the two effectively, the physical parameters of the two antennas were kept the same. The MATLAB source file was extracted from the sensor array analyzer. Figure 3 and Figure 4 shows the MATLAB source code 


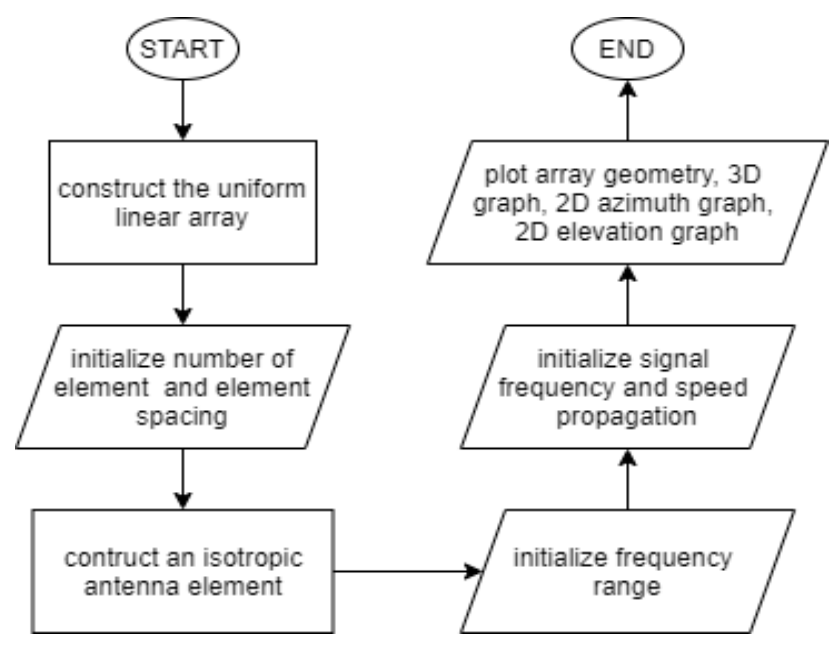

Figure 3. Flowchart of MATLAB source code for omnidirectional antenna

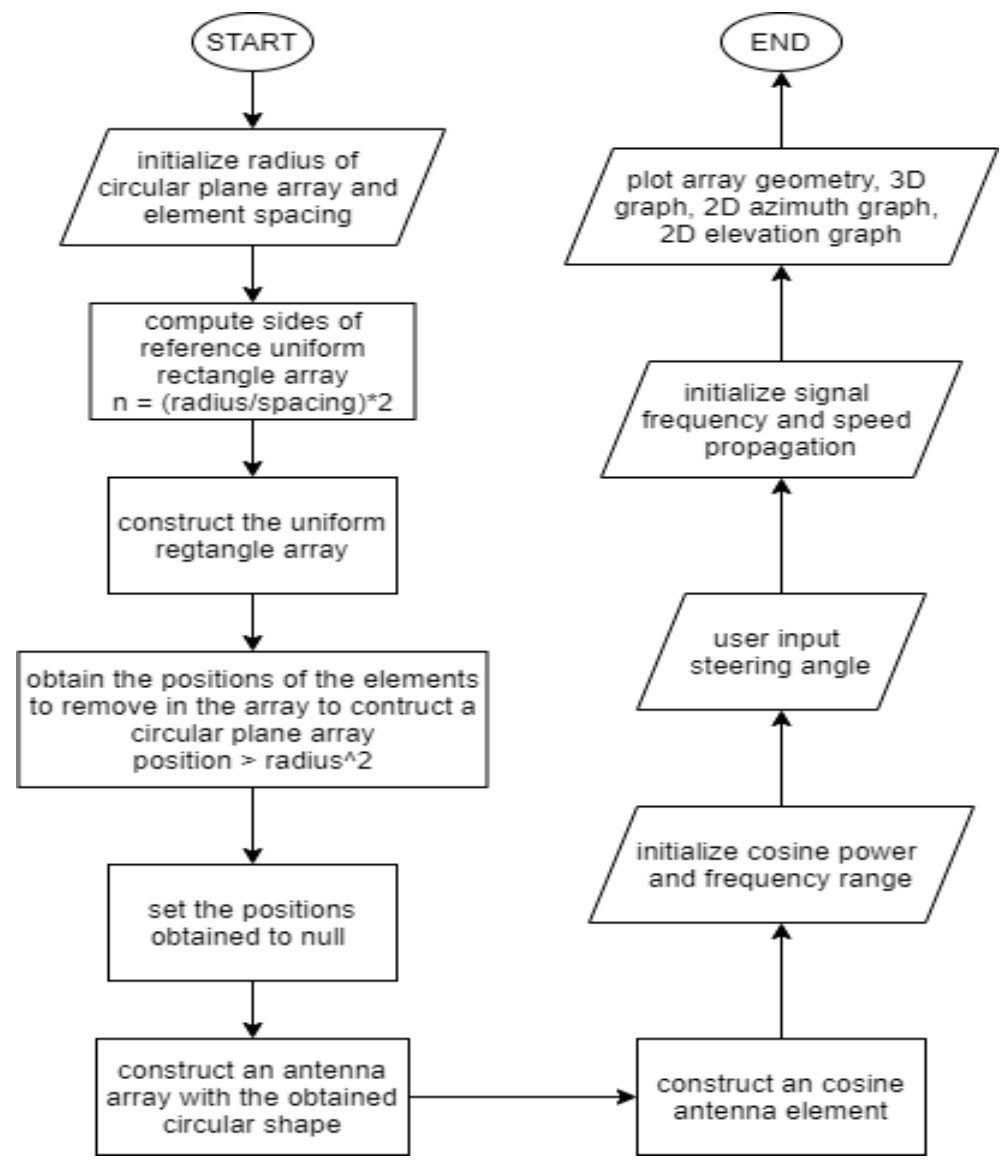

Figure 4. Flowchart of MATLAB source code for adaptive beamforming smart antenna

\section{REVIEW OF RELATED LITERATURE}

Adaptive beamforming smart antenna can be used for indoor positioning systems. The paper "Analysis of Beamforming Antenna for Practical Indoor Location-Tracking Application" done by Kim, S.W., and Choi, D.Y. in 2019 [6] makes use of the beamforming antenna as a single anchor point to receive indoor localization signals. The beamforming antenna used in the experiment is a $1 \times 4$ antenna array connected to a $4 \times 4$ Butler matrix. The function of the Butler matrix is to control the direction of the main lobe. The antenna array inputs the received signal into the Butler matrix and in turn, the Butler matrix feeds a signal with 
varying phases to each element antenna to produce a directed signal. The indoor positioning system was implemented by setting up 10 target locations lined up in a perimeter of a half-circle each separated by an angle of $15 \mathrm{o}$. In the experimentation, it was seen that the higher the signal frequency (between $3 \mathrm{GHz}, 4$ $\mathrm{GHz}$, and $5 \mathrm{GHz}$ ) had a more limited range of angles it could detect due to the beamwidth of the main lobe. The system can detect targets 2 meters away from the anchor point. Additionally, the main lobe of a beamforming antenna can be directed in two directions at the same time, the beamforming antenna in the indoor positioning localization system can also detect multiple targets at a time. The experimentation in the system utilizes the steering angle of the beamforming antenna to detect the location of a target [7].

In high-speed data communications, smart antenna systems are prominently used with various modulation techniques. The article "CL-SA-OFDM: Cross-layer and smart antenna based OFDM system performance enhancement" done by Shivapanchakshari, T.G., and Aravinda, H.S. in 2020 makes use of smart antennas with the modulation technique, orthogonal frequency-division multiplexing (OFDM). With regards to OFDM, adaptive beamforming causes problems in the designs of medium access layers which leads to complications in the adaptive resource allocation mechanism. Various smart antenna mechanisms were used to suit the needs of different users with regards to the quality of service [8].

Smart antenna designs can be utilized to improve transmission rates. The article "Smart antenna design for high-speed moving vehicles with minimum return loss" done by Sarkar, M., Singh, A., Gupta, S., and Hassanien, A. E. in 2020 makes use of a machine learning technique for the design of a smart antenna that can improve transmission rate with a minimized return loss. The experiment suggested a four-element cylindrical antenna array that was wrapped around a cylindrical surface. The bandwidth of the proposed design was set at $200 \mathrm{Mhz}$ with a center frequency of $3.9 \mathrm{GHz}$ and covered the range of 3-3.9 $\mathrm{GHz}$ with a gain of $4.9 \mathrm{dBi}$. The design that was suggested was low profile and was theoretically usable in high-speed avionic applications. The study was made to solve a problem concerning transmission processes in highspeed moving vehicles and the growing concern of power consumption [9].

Adaptive Beamforming may also be used in high-speed mobile communications. The article "Adaptive Beamformers for High-Speed Mobile Communication" done by V. Dakulagi and M. Alagirisamy in 2020 compared various beamforming techniques such as least mean square, recursive least square, and sample matrix inversion to prove that a proposed fast conjugate gradient method was superior to the existing beamforming algorithms and was most suitable for high-speed mobile communication [10].

Previous studies on adaptive beamforming algorithms have also been made. The conference paper "Performance Analysis of Adaptive Beamforming Algorithms" done by P. R. Jeripotula and B. Rajendra Naik in 2018 analyzed two beamforming algorithms which are the least mean square and normalized least mean square algorithms. The paper suggests that the normalized least mean square algorithm converges at a faster rate than the latter. The simulation of both of these algorithms was also conducted in MATLAB [11].

The use of smart antenna systems concerning omnidirectional antennas was conducted with regards to mobile ad hoc networks. The article "A low energy consumption smart antenna adaptive array system for mobile ad hoc networks" by V. Inzillo, F. De Rangoand, A.A. Quintana in 2017 used adaptive array smart antennas in mobile ad hoc networks for the enhancement of properties such as energy management. The experiment proposed an adaptive array smart antenna which would enhance some Ad hoc Distance Vector network protocol features utilizing optimizing the energy consumption. The simulation of the said system used a Least Mean Square algorithm and used the MATLAB software [12].

\section{THEORETICAL CONSIDERATION}

An important toolbox in MATLAB to design and simulate the project is the Phased Array System Toolbox. The phased array system toolbox has built-in commands and applications that contain the algorithm for sensor array systems. The toolbox includes models for transmitter, receivers, and beamforming. The computations for obtaining the radiation patterns were no longer needed since the commands provided by the toolbox only require certain parameters, such as length, number of elements, signal frequency, and such, and the code will generate the radiation patterns needed [13].

The sensor array analyzer application was used to design and simulate the two antennas of the project. An alternative application that can be used is the antenna designer application in the antenna toolbox. To compare the two antennas, the length of the omnidirectional array and the radius of the circular planar array was kept the same. The signal frequency and propagation speed of the antennas were also the same values. According to a study conducted by [14], circular array patterns produce patterns with reduced sidelobe level (SLL) and improved directivity [15]. In the simulation, a uniform circular plane array was used in the design of the adaptive beamforming antenna to enhance the directivity of the antenna. 


\section{DATA AND RESULTS}

The omnidirectional antenna and the adaptive beamforming smart antenna were simulated using the Sensor Array Analyzer in the MATLAB software [16]. Figure 5-10 shows the various radiation pattern.

\subsection{Omnidirectional antenna simulation}

The array directivity and the 3D radiation patterns of both polar and rectangular coordinates are shown. Also shown are the radiation patterns in 2D view.

Array Directivity $=3.01 \mathrm{dBi}$

30 Directivity Pattern

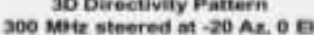

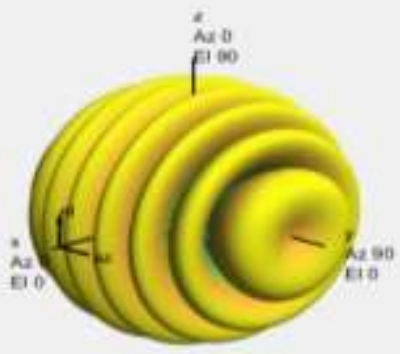

(a)

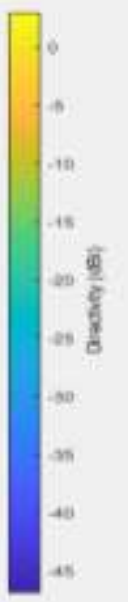

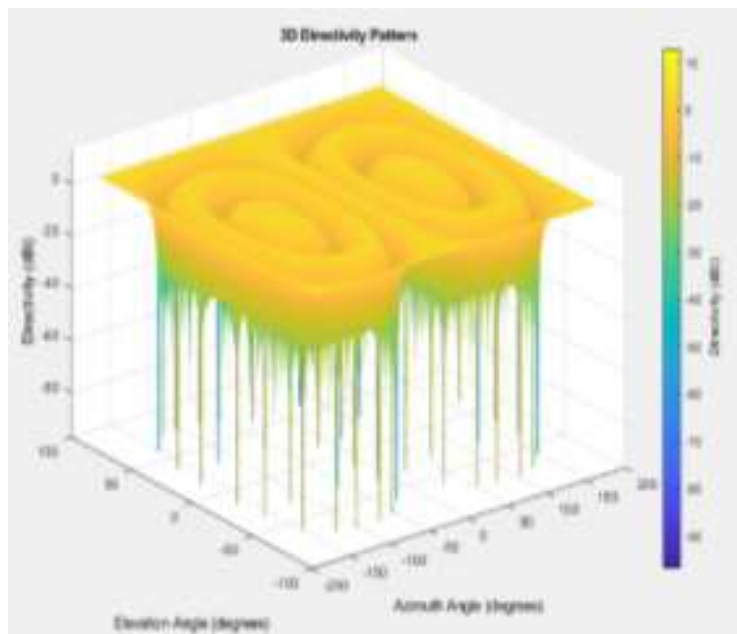

(b)

Figure 5. 3D Radiation pattern in the (a) Polar coordinate and (b) Rectangular coordinate

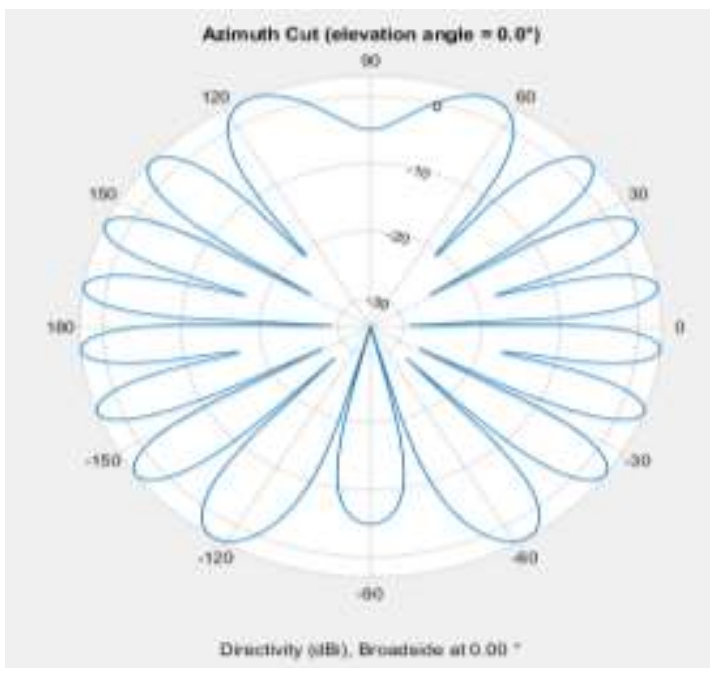

(a)

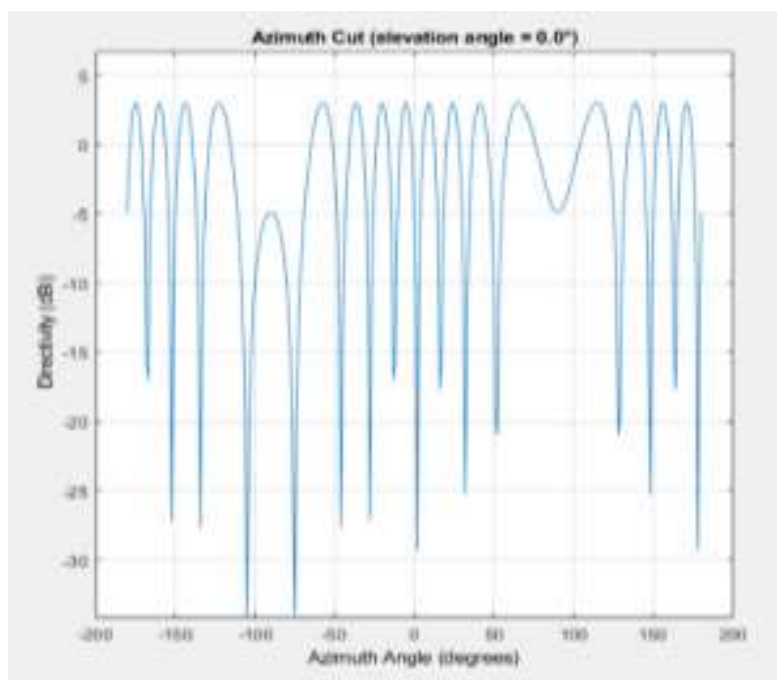

(b)

Figure 6. 2D Radiation pattern on the azimuth plane in the (a) Polar Coordinate and (b) Rectangular coordinate 


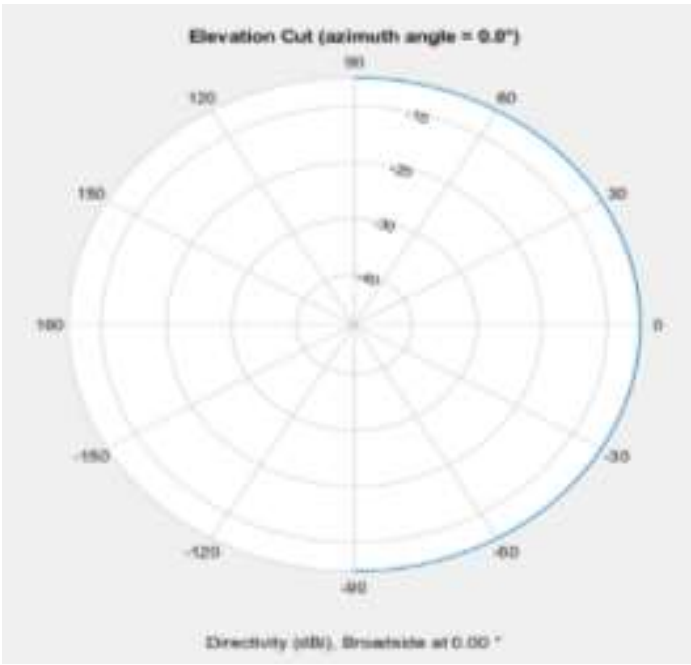

(a)

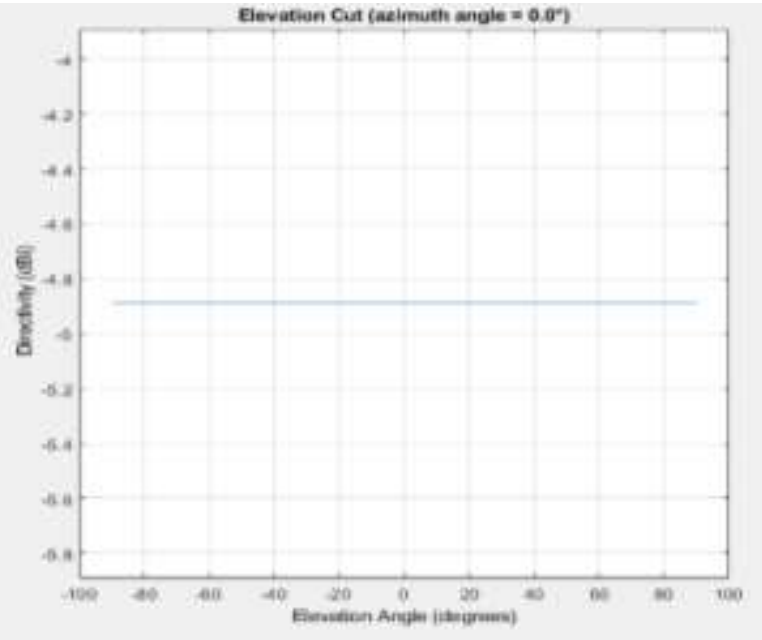

(b)

Figure 7. 2D Radiation pattern on the elevation plane in the (a) Polar coordinate and (b) Rectangular coordinate

\subsection{Adaptive beamforming smart antenna}

The Steering Angle of $-20^{\circ}$ along the azimuth plane was used. The 3D and $2 \mathrm{D}$ patterns in both polar and rectangular coordinates are shown.

Array Directivity $=22.12 \mathrm{dBi}$

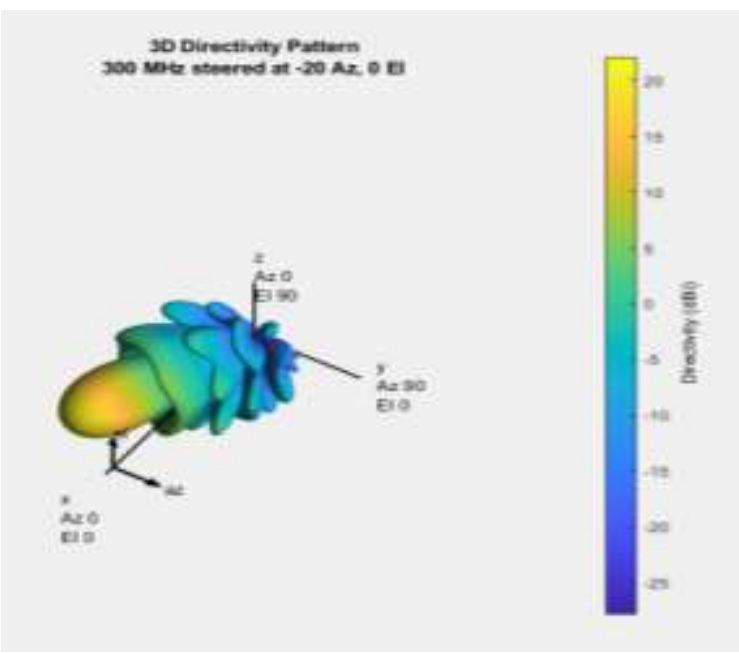

(a)

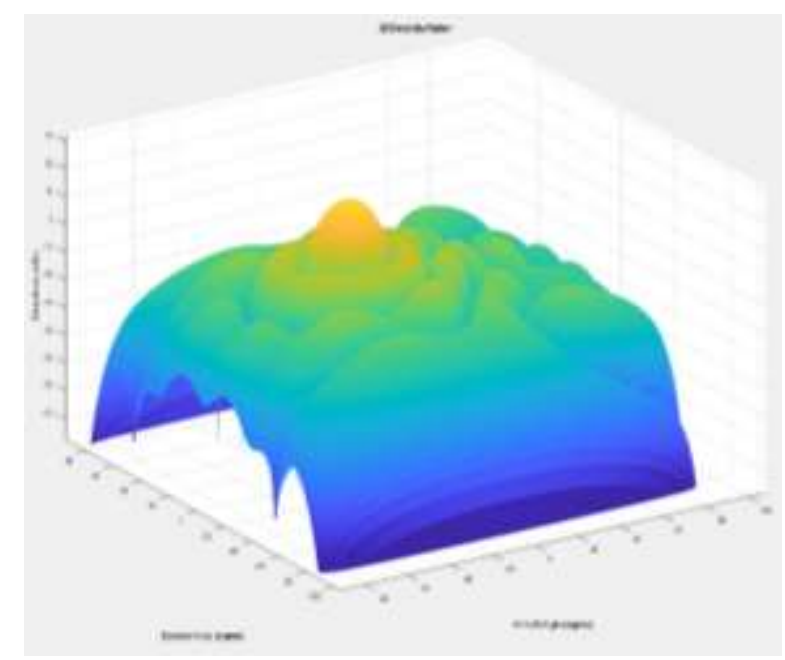

(b)

Figure 8. 3D Radiation pattern in the (a) Polar coordinate and (b) Rectangular coordinate 


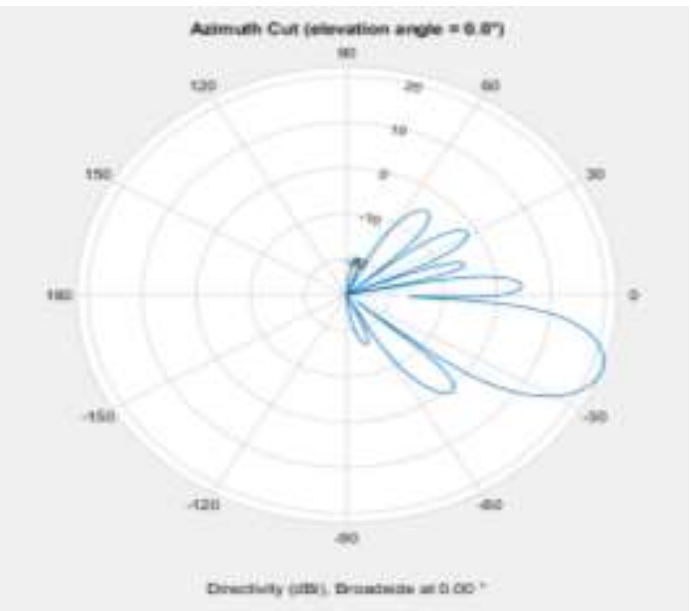

(a)

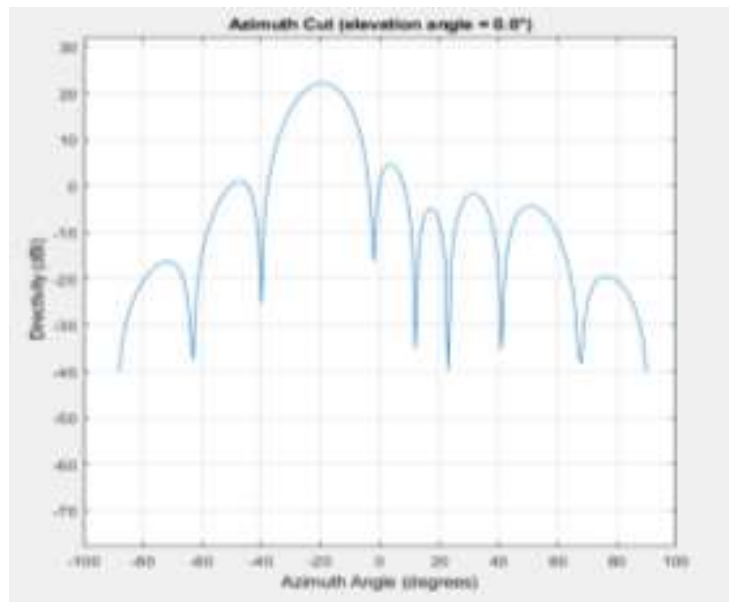

(b)

Figure 9. 2D Radiation pattern on the azimuth plane in the (a) Polar coordinate and (b) Rectangular coordinate

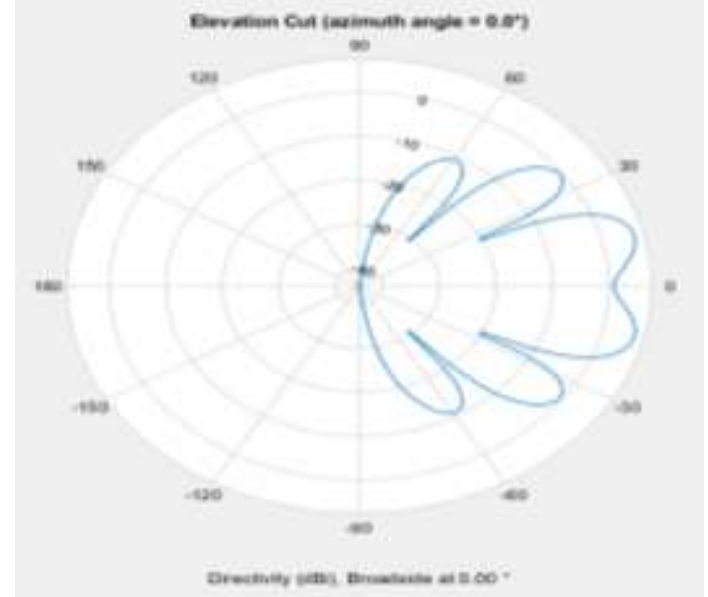

(a)

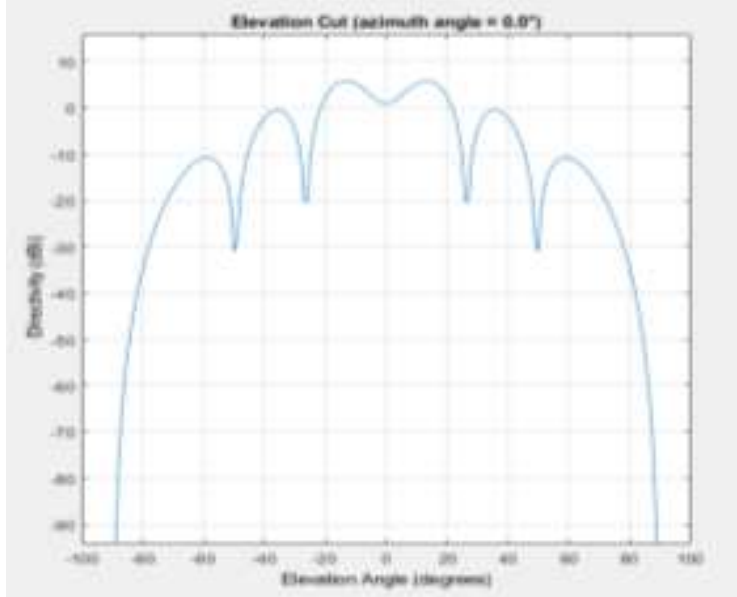

(b)

Figure 10. 2D Radiation pattern on the elevation plane in the (a) Polar coordinate and (b) Rectangular coordinate

\section{ANALYSIS OF DATA}

The antenna directivity gain of an omnidirectional antenna is constant with the element spacing or length between the two poles but inversely proportional to the signal frequency [17-19]. However, as the distance between the two poles decreases or as the signal frequency decreases, the shape of the radiation pattern of the antenna is closer to an isotropic radiator. The antenna diversity of the adaptive beamforming smart antenna varies differently on the changing radius, element spacing, and frequency signal.

As the radius of the circular array decreases, the antenna directivity gain decreases and the beamwidth of the radiation pattern widens. As the signal frequency decreases, the beamwidth widens, and consequently, the antenna directivity decreases. As the spacing increases, the sidelobes get stronger. Consequently, as the spacing decreases and the direction of the radiated energy is focused on one direction, the gain increases, and the sidelobes get suppressed. The result concludes that as the number of element arrays increases, the antenna can further focus the radiated energy in a specific direction. The smart antenna can propagate signals that reach longer distances [20-22].

When the parameters of the two antennas are matched to compare their directivity, it can be seen that the gain of the adaptive beamforming smart antenna is higher than the gain of the omnidirectional antenna by $19.11 \mathrm{dBi}$. It confirms the concept that a directional antenna radiates a stronger signal than an omnidirectional antenna [23-25]. The physical implementation of the simulation would consider the cost as a 
limiting factor when using the adaptive beamforming smart antenna. The circuitry of an omnidirectional antenna is simpler relative to the directional antenna. This is reflected in the MATLAB source codes of the two antennas. The circular planar array was first set up before the simulation of the antenna itself. The run time of the directional antenna is longer than the omnidirectional.

\section{CONCLUSION}

The application Sensor Array Analyzer from the MATLAB software provided the researchers with ease in design and simulation of the omnidirectional antenna and the adaptive beamforming smart antenna. The phased array toolbox provided the algorithms needed to simulate the two antennas. The computation of the radiation intensity to graph the radiation pattern is done by the toolbox. The model of the adaptive beamforming smart antenna was done in the application. A circular planar array geometry was chosen for the adaptive antenna due to its ability to suppress sidelobes and improve directivity.

The simulation presented that the circular planar geometry was effective in the directivity of the antenna. Other variations of the parameters were also explored with its effects were observed. The omnidirectional antenna with similar parameters was also simulated. The variations of the parameters were also explored with its effects were observed. The radiation patterns of the two antennas were observed along with its gain for an isotropic antenna. The results of the simulation confirmed that the adaptive beamforming smart antenna has a higher gain compared to the omnidirectional antenna. The stronger gain of an antenna means that it could travel a greater distance. The steering angle leads the angle of direction of the radiated energy. It causes the shift in the phases of the signals of the elements in the array of the antenna.

The simulation also illustrated the complexity of the circuit of the two antennas. In as simple as the run time of the simulation, it can be seen that the run time of the omnidirectional antenna is faster than the adaptive beamforming smart antenna. The software generated the output of the adaptive antenna at a relatively much longer time due to the complexity of the algorithm involved. This would be reflected in the cost of the two antennas. Due to its complexity, adaptive antennas are more expensive than omnidirectional antennas. However, in certain applications, the complexity and cost of a directional antenna can be overlooked.

\section{REFERENCES}

[1] B. Kumoro and Y. Mukhlis, "Dipole antenna design with CPS matching for RFID application," Journal of Physics: Conference Series, vol. 1175, no. 1, 2019.

[2] M. Wnuk, "Antenna radiation patterns indication on the basic measurement of field radiation in the near zone," WIT Transactions on Modelling and Simulation, vol. 48, pp. 191-202, 2009.

[3] P. Hazdra, M. Capek, M. Masek, and T. Lonsky, "An introduction to the source concept for antennas," Radioengineering, vol. 25, no. 1, pp. 12-17, 2016.

[4] D. Savitri, A. Azzahra, P. Wulandari, and S. Rahmatia, "Comparison between hollow aluminum yagi-uda and dipole antenna for indoor antenna TV," IOP Conference Series: Materials Science and Engineering, vol. 453, no. $1,2018$.

[5] F. Ali, A. Urban, and M. Vossiek, "A high resolution 2D omnidirectional synthetic aperture radar scanner at K band," the European Microwave Week 2010, EuMW2010: Connecting the World, Conference Proceedings European Radar Conference, EuRAD 2010, pp. 503-506, 2010.

[6] A. Innok, P. Uthansakul, and M. Uthansakul, "Angular beamforming technique for MIMO beamforming system," 2012 9th International Conference on Electrical Engineering/Electronics, Computer, Telecommunications, and Information Technology, ECTI-CON, 2012.

[7] W. Li, Y. Zhao, and Q. Ye, "Semi-virtual antenna array beamforming method," Progress in Electromagnetics Research Letters, vol. 68, pp. 33-37, 2017.

[8] S. Kim and D. Choi, "Analysis of beamforming antenna for practical indoor location-tracking application," Sensors (Switzerland), vol. 19, no. 14, 2019.

[9] T. Shivapanchakshari and H. Aravinda, "CL-SA-OFDM: Cross-layer and smart antenna based OFDM system performance enhancement," International Journal of Electrical and Computer Engineering, vol. 10, no. 5, pp. 4663-4670, 2020.

[10] M. Sarkar, A. Singh, S. Gupta, and A. Hassanien, "Smart antenna design for high-speed moving vehicles with minimum return loss," International Journal of Communication Systems, vol. 33, no. 11, 2020.

[11] V. Dakulagi and M. Alagirisamy, "Adaptive beamformers for high-Speed Mobile communication," Wireless Personal Communications, vol. 113, no. 4, pp. 1691-1707, 2020.

[12] P. Jeripotula and B. Rajendra Naik, "Performance analysis of adaptive beamforming algorithms," 2018 International Conference on Circuits and Systems in Digital Enterprise Technology, ICCSDET, 2018,

[13] V. Inzillo, F. De Rango, and A. Quintana, "A low energy consumption smart antenna adaptive array system for mobile ad hoc networks," International Journal of Computing, vol. 16, no. 3, pp. 124-132, 2017. 
[14] V. Chakravarthy Vedula, S. Chowdary Paladuga, and M. Rao Prithvi, "Synthesis of Circular Array Antenna for Sidelobe Level and Aperture Size Control Using Flower Pollination Algorithm," International Journal of Antennas and Propagation, vol. 2015, 2015.

[15] A. Africa, A. Alejo, G. Bulaong, S. Santos, and J. Uy, "Effect of dielectric substrate on dipole antenna directivity," International Journal of Emerging Trends in Engineering Research, vol. 7, no. 8, pp. 170-177, 2019.

[16] MathWorks, MATLAB, [Online] Available: https://www.mathworks.com/products/matlab.html, 2015.

[17] M. Said, M. Misran, Z. Zakaria, M. Zin, and L. Muhammad, "Design of a circularly polarized antenna at $2.45 \mathrm{ghz}$ with harmonic suppression for material characterization," International Journal of Emerging Trends in Engineering Research, vol. 8, no. 8, pp. 4420-4425, 2020.

[18] A. Sudhakar, M. Sunil Prakash, and M. Satyanarayana, "Development of offset microstrip line fed patch antenna for 4.9 ghz public safety wlan," International Journal of Emerging Trends in Engineering Research, vol. 8, no. 8, pp. 4213-4219, 2020.

[19] M. Misran, M. Said, A. Salleh, and R. Ramlee, "Ultra-wideband antenna with Y-shape defected ground structure," International Journal of Emerging Trends in Engineering Research, vol. 8, no. 7, pp. 3588-3593, 2020.

[20] C. Amarnatha Sarma, S. Inthiyaz, and B. Madhav, "Effect of ground etching, inset feed and substrate height on elliptically shaped patch antenna," International Journal of Emerging Trends in Engineering Research, vol. 8, no. 7, pp. 3145-3149, 2020.

[21] A. Salleh, M. Aziz, Z. Zakaria, M. Misran, and N. Hashim, "Electromagnetic radiation effect of action potential based on different antenna position in homogenous human arm flat and cylindrical shape model using ultra wideband coplanar stripline-fed antenna," International Journal of Emerging Trends in Engineering Research, vol. 8, no. 6, pp. 2557-2562, 2020.

[22] V. Marwaha, H. Saini, and D. Arora, "A j-shaped element planar inverted-f mimo antenna for $4 \mathrm{~g} / 5 \mathrm{~g}$ communication," International Journal of Emerging Trends in Engineering Research, vol. 8, no. 2, pp. 602-605, 2020.

[23] K. Krishna, H. Khan, and K. Naik, "A compact rectangular shaped dipole array slot microstrip antenna with DGS for multiband applications," International Journal of Emerging Trends in Engineering Research, vol. 8, no. 2, pp. 408-413, 2020.

[24] S. Venkata Rama Rao, A. Mallikarjuna Prasad, and C. Rani, "Antenna array pattern nulling by phase perturbations using modified differential evolution algorithm," International Journal of Emerging Trends in Engineering Research, vol. 7, no. 9, pp. 256-261, 2019.

[25] A. Fadl, M. Ihedrane, and S. Bri, "Estimation direction arrival of smart antenna," International Journal of Emerging Trends in Engineering Research, vol. 8, no. 4, pp. 1051-1055, 2020.

\section{BIOGRAPHIES OF AUTHORS}

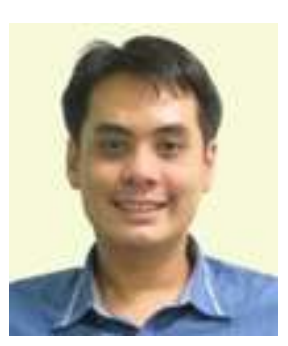

Aaron Don M. Africa is a researcher in the field of Communications Network Engineering. He is one of the most promising researchers in that area. His area of expertise are communications network engineering, expert systems, control systems, network design, and optimization. specifically, he deals with the optimization of communication systems to make them more efficient in functionality. This is for these systems to adapt effectively in the industry. He creates simulation models to replicate different scenarios in network design.

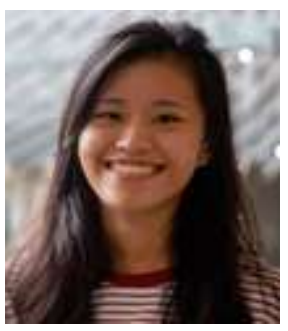

Rica Rizabel M. Tagabuhin is from Manila, Philippines. She is a student of the B.S. degree in Electronics and Communications Engineering (ECE) from De La Salle University. She is a member of the Electronics and Communications Engineering society (ECES) and her research interests include data communications.

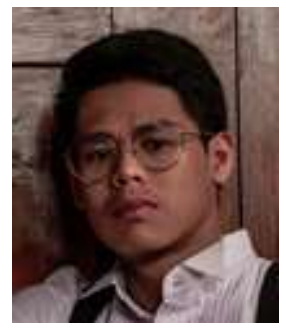

Jan Jayson S.D. Tirados is from Manila, Philippines. He is a student of the B.S. degree in Electronics and Communications Engineering (ECE) from De La Salle University. He is a member of the Electronics and Communications Engineering Society (ECES) and his research interests include antenna design. 